\title{
High efficiency grating coupler between silicon-on-insulator waveguides and perfectly vertical optical fibers
}

\author{
Günther Roelkens, * Dries Van Thourhout, and Roel Baets \\ Photonics Research Group, Ghent University-IMEC, Sint-Pietersnieuwstraat 41, B-900o Ghent, Belgium \\ *Corresponding author: gunther.roelkens@intec.ugent.be
}

Received February 8, 2007; revised March 26, 2007; accepted March 27, 2007; posted March 29, 2007 (Doc. ID 79941); published May 4, 2007

A high-efficiency waveguide-to-fiber grating coupler for silicon-on-insulator waveguides was designed. Perfectly vertical fiber coupling is achieved by using an asymmetric grating structure to suppress the secondorder Bragg reflection from the grating. The ability to use a perfectly vertical positioned optical fiber simplifies the packaging of the photonic integrated circuit. A coupling efficiency of $80 \%$ at a wavelength of $1.55 \mu \mathrm{m}$ is obtained. (C) 2007 Optical Society of America

OCIS codes: $050.2770,130.0130$.

Silicon-on-insulator (SOI) technology is emerging as a promising platform for integrated optics due to the high refractive index contrast between the silicon core and the oxide cladding $(\Delta n \cong 2)$. This enables high-density integrated optical circuits, which can be fabricated by standard complementary metal-oxide semiconductor (CMOS) technology [1]. One of the drawbacks of the high refractive index contrast is the large mismatch in mode size and mode shape between the fundamental mode of the SOI waveguide and the mode of the optical fiber, making efficient coupling of light from waveguide to fiber an important issue. In the literature, one-dimensional [2] and two-dimensional [3] grating structures were proposed to tackle this problem. These grating couplers have the advantage of not having to cleave the devices for optical coupling, which enables wafer-scale testing of the integrated circuits and holds the promise of low-cost packaging. Furthermore, they are very compact and have a large optical bandwidth. Although one-dimensional structures are polarization dependent, a polarization diversity scheme based on a two-dimensional grating coupler can be used in practical applications [3]. To obtain a high coupling efficiency, different strategies can be followed. By including a bottom mirror to redirect the downward diffracted light, a high coupling efficiency can be obtained. This can be a metallic mirror [4] or a distributed Bragg reflector-type mirror [5]. Another way to improve the coupling efficiency is to optimize the design parameters of the grating unit cell while tailoring the pitch and duty cycle of the individual grating periods [6]. However, all these designs rely on slightly tilting the optical fiber with respect to the vertical axis. This is necessary to avoid a large second-order Bragg reflection back into the SOI waveguide in the case of perfectly vertical coupling, which dramatically reduces the fiber coupling efficiency. This requirement has important consequences for practical applications, which would imply angled polishing of the fiber ferrule and mounting of the ferrule under an angle with respect to the photonic integrated circuit normal direction [7]. To avoid this costly complication in the packaging of the photonic integrated circuit, the use of a slanted grating coupler was proposed to obtain high coupling efficiency to a vertically positioned optical fiber [8]. The technology required for fabrication of these slanted slits is, however, very complex and therefore only suitable for prototyping. In this Letter, the possibility for perfectly vertical coupling in an efficient way is assessed by designing an asymmetric grating structure based on vertically etched slits to avoid the large secondorder Bragg reflection such that the fabrication process remains CMOS compatible. The proposed grating structure for perfectly vertical fiber coupling is shown in Fig. 1. The waveguide-to-fiber grating coupler consists of a grating that predominantly diffracts the light upward and an additional slit etched in the $220 \mathrm{~nm}$ thick silicon input waveguide, which acts as a partially reflecting mirror, to achieve destructive interference of the second-order Bragg reflection. In principle, the width and etch depth of the slits, both in the input waveguide and in the diffraction grating region, can be individually optimized.

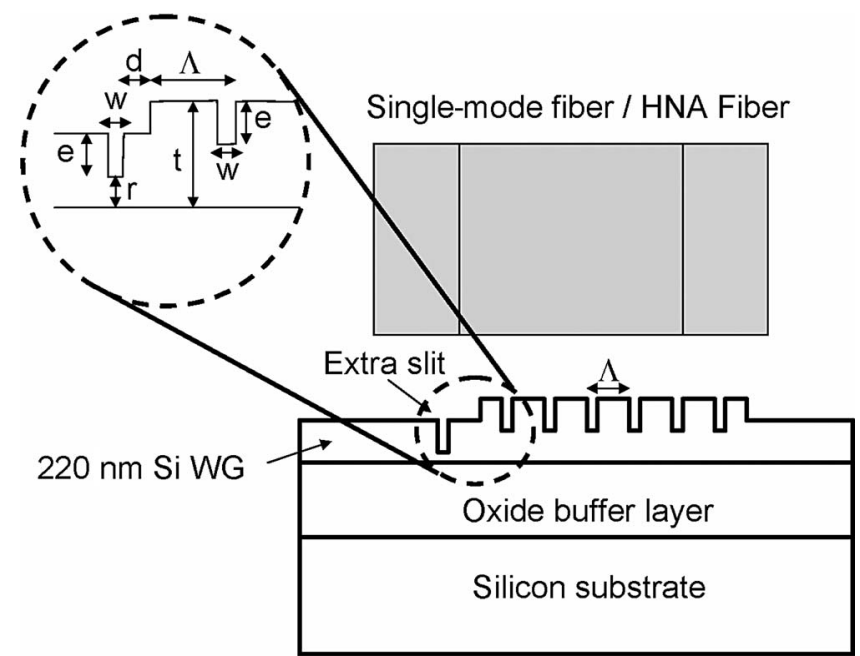

Fig. 1. Layout of the proposed grating coupler structure for efficient coupling from an SOI waveguide to a vertically positioned single-mode fiber. 
However, from a fabrication point of view, the dimensions of these slits should be identical, as different etch rates can be expected for slits of different widths.

The behavior of the etched slit in the $220 \mathrm{~nm}$ thick silicon waveguide layer as a partially reflecting mirror was assessed by calculating the power reflection and scattered optical power as a function of the slit etch depth, using the slit width $w$ as a parameter. Results are shown in Fig. 2 for three different etch depths, corresponding to $r=0$ (etched through the silicon waveguide layer), $r=25 \mathrm{~nm}$ and $r=50 \mathrm{~nm}$. CAMFR [9], a two-dimensional eigenmode expansion tool with perfectly matched layers absorbing boundary conditions, was used for the simulations. A wavelength of $1.55 \mu \mathrm{m}$ and TE polarization is assumed. From this simulation, it is clear that the completely etched-through slit offers the largest reflection for a given acceptable scattering loss. Therefore, in the subsequent analysis, the slit is assumed to be etched completely through the $220 \mathrm{~nm}$ silicon waveguide layer. This assumption also fixes the slit etch depth $e$ in the uniform grating structure to $220 \mathrm{~nm}$, for ease of fabrication. To keep the scattering losses low while maintaining a sufficient reflection, the minimum definable slit width using CMOS $248 \mathrm{~nm}$ deep UV lithography of $160 \mathrm{~nm}$ was chosen for the design. This then also fixes the slit width $w$ of the diffractive grating structure to $160 \mathrm{~nm}$.

In a second part of the optimization, the thickness of the silicon layer stack in the diffractive grating region and the period of the grating is optimized in order to achieve diffraction in the vertical direction at $1.55 \mu \mathrm{m}$ while maximizing the directionality (the ratio of upward diffracted optical power to the total diffracted power) of the grating at this wavelength. This can be done by evaluating the stationary leaky Bloch mode at the $\Gamma$ point supported by the periodic grating structure and assessing the directionality of this leaky mode. Optimal directionality and vertical coupling at $1.55 \mu \mathrm{m}$ was obtained for a silicon layer thickness of $370 \mathrm{~nm}$ and a grating period $\Lambda$ of $560 \mathrm{~nm}$. The electric field of the vertically leaking Bloch mode with optimized directionality is plotted in Fig. 3. A directionality higher than $80 \%$ is obtained.

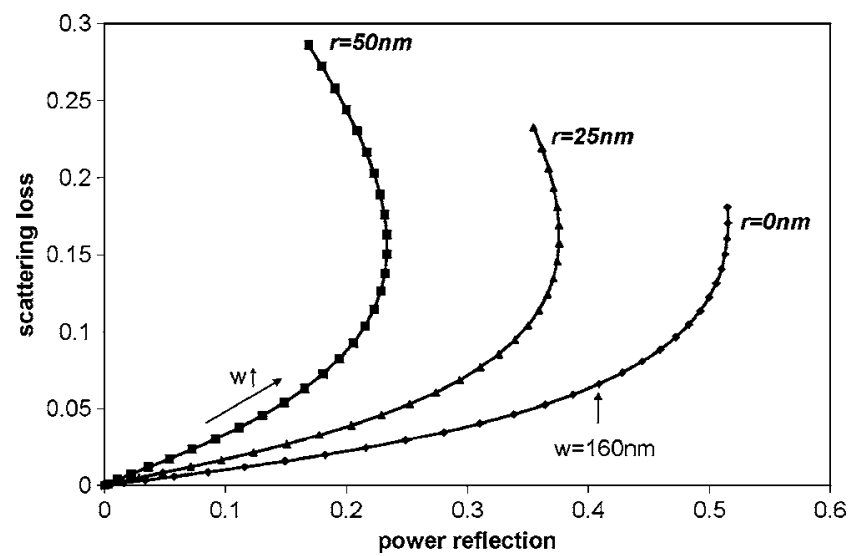

Fig. 2. Scattering loss of a single slit in a $220 \mathrm{~nm}$ SOI waveguide as a function of the power reflection for various slit widths and depths.

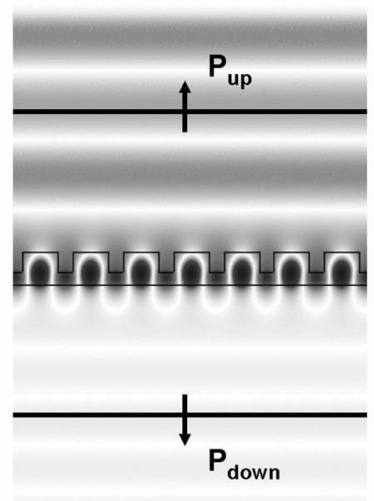

\section{Directionality $=P_{\text {up }} /\left(P_{\text {up }}+P_{\text {down }}\right)$}

Fig. 3. Electric field of the vertically leaky Bloch mode with optimized directionality.

The influence of the distance $d$ (between the additional slit in the input waveguide and the diffraction grating) on the power reflection into the SOI waveguide, when exciting the diffractive grating structure with the fundamental SOI waveguide mode from the left-hand side, was assessed. For an optimal distance $d$ of $0.13 \mu \mathrm{m}$, a nearly perfect destructive interference of the second-order Bragg reflection is achieved (power reflection below 1\%). To appreciate the influence of the additional slit, the power reflection in the case when no additional slit in the input waveguide is used, was calculated to be $55 \%$. To calculate the coupling efficiency to fiber, the grating structure is excited by the power normalized fundamental waveguide mode, and the diffracted electrical field pattern $\mathbf{E}_{\text {diff }}$ in the air cladding at a certain height above the grating is calculated. The coupling efficiency $\eta$ is then calculated by evaluating the overlap integral:

$$
\eta=\left|\int \mathbf{E}_{\text {diff }} \times \mathbf{H}_{f i b}^{*} \cdot \mathbf{d} \mathbf{S}\right|^{2},
$$

in which $\mathbf{H}_{\text {fib }}$ represents the magnetic field distribution of the power normalized Gaussian fiber waveguide mode. This analysis neglects the reflection at the fiber-air interface. The overlap integral is evaluated for different fiber mode field diameters [(MFDs), the $1 / e^{2}$ intensity width] and for different positions of the optical fiber with respect to the grating, resulting in the optimal fiber coupling efficiency. To correct this two-dimensional fiber coupling efficiency for the mismatch between the lateral Gaussian fiber mode profile and the SOI waveguide mode profile, the overlap integral between these lateral mode profiles was used as a correction factor [5]. An optimal coupling efficiency of $66 \%$ is obtained for an MFD of $4 \mu \mathrm{m}$, while $50 \%$ coupling efficiency is obtained for a standard single-mode fiber with an MFD of $9 \mu \mathrm{m}$. In practical applications, reduced mode field diameters can be obtained by splicing a high numerical aperture fiber to a single-mode fiber with low optical loss at the splice [10].

To further increase the fiber coupling efficiency, a nonuniform grating structure can be applied, as this 


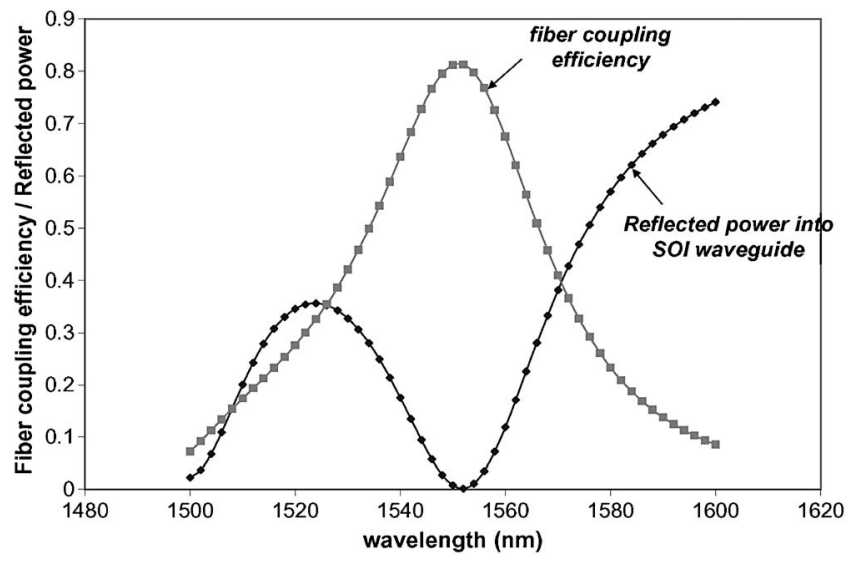

Fig. 4. Fiber coupling efficiency and reflection back into the SOI waveguide for a nonuniform grating structure, using a $4 \mu \mathrm{m}$ mode field diameter optical fiber.

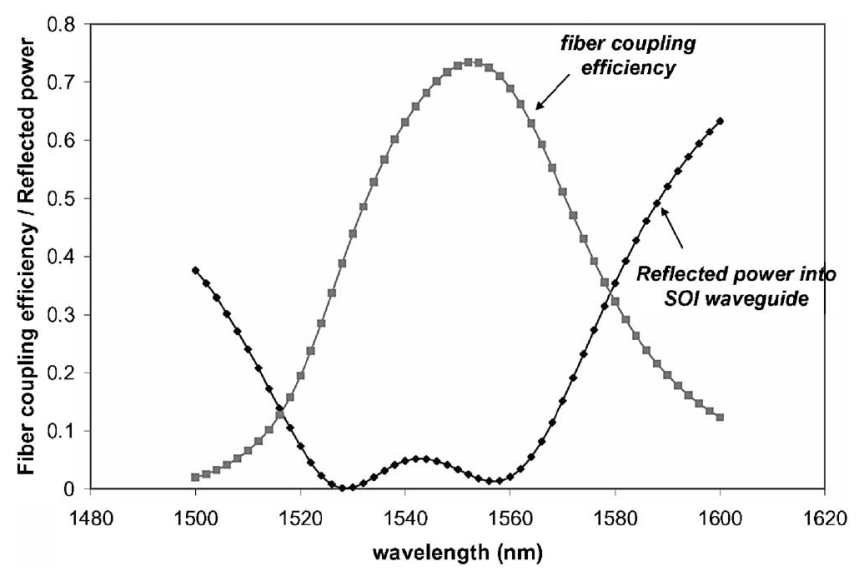

Fig. 5. Fiber coupling efficiency and reflection back into the SOI waveguide for a nonuniform double slit configuration, using a $4 \mu \mathrm{m}$ mode field diameter optical fiber.

allows achieving a better match between the diffracted field profile and the Gaussian optical fiber mode. As the width of the slits has to be kept identical throughout the grating structure as discussed above, only the distance between subsequent slits can be varied. A genetic algorithm optimization was performed to maximize the coupling efficiency to an optical fiber with a $4 \mu \mathrm{m}$ MFD. The fiber coupling efficiency increased from $65 \%$ for the uniform grating structure to $80 \%$ for the nonuniform grating structure. As the minimum pitch in the optimized structure is $530 \mathrm{~nm}$, these nonuniform grating structures can be fabricated using standard CMOS technology. The fiber coupling efficiency spectrum for this nonuniform grating structure is plotted in Fig. 4. The power reflection back into the SOI waveguide, when the grating is excited from the left-hand side waveguide, is also plotted.

As can be seen from this figure, the power reflection back into the SOI waveguide is minimal at
$1.55 \mu \mathrm{m}$, but rises quickly for a slight wavelength detuning. This limits the effective bandwidth of the grating coupler structure, as substantial reflection back into the SOI waveguide has to be avoided. To extend the effective bandwidth of the grating coupler structure, multiple additional slits in the $220 \mathrm{~nm}$ SOI waveguide layer can be used to achieve a low-power reflection over a broader wavelength range. Optimization of a nonuniform grating structure, with two slits etched in the standard $220 \mathrm{~nm}$ silicon waveguide, was carried out using a genetic optimization algorithm as well. A high numerical aperture fiber with a MFD of $4 \mu \mathrm{m}$ was assumed. The average fiber coupling efficiency at 1.54 and $1.56 \mu \mathrm{m}$ was maximized, while minimizing the average power reflection at these wavelengths. The resulting fiber coupling efficiency spectrum and power reflection spectrum are plotted in Fig. 5. As can be seen from this simulation, the effective bandwidth of the device has improved, at the expense of the maximal fiber coupling efficiency compared with the single slit configuration (due to the additional scattering at the extra slit).

To conclude, a high efficiency, broadband grating coupler for perfectly vertical fiber coupling was designed by making the grating structure asymmetric. This was achieved by defining additional slits in the input waveguide. A coupling efficiency of $80 \%$ can be obtained for a grating structure that can be defined using standard CMOS processing techniques, which is interesting for a mass market application.

\section{References}

1. W. Bogaerts, R. Baets, P. Dumon, V. Wiaux, S. Beckx, D. Taillaert, B. Luyssaert, J. Van Campenhout, P. Bienstman, and D. Van Thourhout, J. Lightwave Technol. 23, 401 (2005).

2. D. Taillaert, W. Bogaerts, P. Bienstman, T. F. Krauss, P. Van Daele, I. Moerman, S. Verstuyft, K. De Mesel, and R. Baets, IEEE J. Quantum Electron. 38, 949 (2002).

3. D. Taillaert, H. Chong, P. I. Borel, L. H. Frandsen, R. M. De La Rue, and R. Baets, IEEE Photon. Technol. Lett. 15, 1249 (2003).

4. F. Van Laere, G. Roelkens, J. Schrauwen, D. Taillaert, P. Dumon, W. Bogaerts, D. Van Thourhout, and R. Baets, Presented at the Conference on Optical Fiber Communication (IEEE, 2006), paper PDP15.

5. D. Taillaert, P. Bienstman, and R. Baets, Opt. Lett. 29, 2749 (2004).

6. G. Roelkens, D. Van Thourhout, and R. Baets, Opt. Express 14, 11622 (2006).

7. P. Dumon, W. Bogaerts, D. van Thourhout, D. Taillaert, R. Baets, J. Wouters, S. Beckx, and P. Jaenen, Opt. Express 14, 664 (2006).

8. B. Wang, J. H. Jiang, and G. P. Nordin, IEEE Photon. Technol. Lett. 17, 1884 (2005).

9. P. Bienstman and R. Baets, IEE Proc. Optoelectron. 149, 161 (2002).

10. J. Harper, C. Botham, and S. Hornung, Electron. Lett. 24, 245 (1988). 